\title{
Desempenho fisiológico de sementes de Zea mays matricondicionadas em diferentes temperaturas
}

Alexandre Hack Porto, Carlos Kosera Neto, Jean Carlo Possenti, Edgar de Souza Vismara, Lilian de Souza Vismara, Thayllane de Campos Siega

Universidade Tecnológica Federal do Paraná - UTFPR, PR. E-mail: alexandrehack@gmail.com

\section{Resumo}

O uso de sementes de alta qualidade reflete diretamente sobre o resultado final do plantio, estabelecimento da cultura e produtividade. A velocidade de entrada de água nas sementes e a temperatura são fatores cruciais no processo germinativo. Por isso, objetivou-se quantificar o ganho de água de sementes de milho submetidas a matricondicionamento em quatro temperaturas $(10 ; 15 ; 20$ e 25 ${ }^{\circ} \mathrm{C}$ ), bem como, avaliar a qualidade fisiológica destas sementes após a pré-hidratação controlada. Foram usadas sementes $\mathrm{C} 1$, sem tratamento químico, de cultivares de milho híbrido. Em um primeiro momento, determinou-se a curva de embebição de água a partir de oito repetições de duzentas sementes via análise de regressão do teor de água absorvido em função dos intervalos de tempo de 2; 4; 6; 10;14; 18 e 24 horas. Em sequência, vários testes foram realizados para identificar a qualidade fisiológica das sementes. Ao final das 24 horas de avaliação, ambos os lotes apresentaram maior porcentagem de umidade quando matricondicionadas à temperatura de $25^{\circ} \mathrm{C}$. A qualidade fisiológica das sementes de milho foi afetada pela embebição controlada e maiores temperaturas proporcionam a expressão de maior vigor nas plântulas e inferindo que a técnica de matricondicionamento se mostrou eficaz para realizar o condicionamento osmótico de sementes desta espécie.

Palavras-chave: milho; pré-hidratação; condicionamento osmótico; desempenho fisiológico; vigor.

\section{Physiological performance of Zea mays seeds matriconditioned at different temperatures}

\begin{abstract}
The use of high quality seeds reflects directly on crop establishment and productivity. The speed of seed water intake, as well as, temperature during this process is the main factor to have success during germination. The objective of this study was quantifying water uptake from corn seeds during matriconditioning under four temperatures $\left(10,15,20\right.$ and $25^{\circ} \mathrm{C}$ ) and seeds without treatment (control) also evaluate physiological quality of those. $\mathrm{C} 1$ seed, without chemical treatment, from two different lots of $30 \mathrm{~F} 53 \mathrm{YH}$ hybrid corn was used. The water content curve was determined from eight replication, of two hundred seeds each, by weighing in intervals of $2 ; 4 ; 6 ; 10 ; 14 ; 18$; and 24 hours of soaking. In sequence various tests aiming identify physiological seeds quality was developed. With 24-hour evaluation, both group of seeds had higher moisture content when matriconditioned at $25^{\circ} \mathrm{C}$. The quality of corn seeds was affected by matriconditioning and the higher imbibition temperatures provided the expression of greater vigor in corn seedlings, being useful for this specie.
\end{abstract}

Keywords: corn; pre-hydration; seed conditioning; physiological development; vigor.

\section{Introdução}

O milho (Zea mays L.) apresenta importância econômica, sendo cultivado em diversas partes do mundo e amplamente utilizado para alimentação ou matéria prima de diversas indústrias (TIAN et al., 2014).

A utilização de sementes de alta qualidade é fator primordial para qualquer 
cultura, já que assegura o estabelecimento e população adequada, frente às possíveis variações de condições ambientais durante a emergência (SCHEEREN et al., 2010). As estruturas das sementes absorvem água em velocidades diferentes e são dependentes de fatores como espécie, disponibilidade hídrica, área de contato e temperatura (CARVALHO; NAKAGAWA, 2000).

A funcionalidade celular nos organismos eucariontes depende do sistema eficiente de compartimentalização celular, provido por endomembranas, portanto, para que as células mantenham sua funcionalidade metabólica é imprescindível que suas membranas apresentemse íntegras (COSTA et al., 2008). A temperatura é fator crucial nesse processo, já que afeta a velocidade e uniformidade de germinação (MARTINS et al., 2008; PASSOS et al., 2008).

$\mathrm{O}$ uso de testes de vigor associados aos de germinação e emergência são utilizados rotineiramente pelas empresas produtoras de sementes, tendo se intensificado dentro do controle interno de qualidade, a fim de estimar o potencial em campo, tanto em condições favoráveis como adversas (GRZYBOWSKI et al., 2015). Contudo, em função do processo de embebição das sementes ser fator que pode alterar o funcionamento das membranas celulares, os resultados dos testes de vigor necessitam serem vistos com ressalva. Pois o controle da velocidade de hidratação das sementes podem resultar em seu maior ou menor desempenho.

O componente fisiológico da qualidade de sementes é objeto de inúmeros estudos e podem influenciar respostas ao desempenho fisiológico (PALLAORO et al., 2016), em função das sementes estarem sujeitas à gama de mudanças degenerativas após a maturidade (FREITAS; NASCIMENTO, 2006). Para determinação do potencial fisiológico dos lotes de sementes são feitos testes que podem fornecer informações sobre o resultado da interação entre o armazenamento e vigor das mesmas (DIAS et al., 2019).

Dentre as técnicas de condicionamento ou pré-tratamento de sementes que se objetiva aumentar a taxa, uniformidade de germinação e o vigor, o matricondicionamento pode ser eficaz, é de fácil aplicação pela utilização de matrizes sólidos com alta forças capilares de adsorção de água (KHAN et al., 1990). As matrizes sólidas mais utilizadas são vermiculita, argila expandida calcinada, gel de polipropionato sódico, silicato cálcio sintético, carvão vegetal e serragens (KHAN et al., 1992).

Dessa forma, objetivou-se quantificar o ganho de água em sementes de milho cultivares 30F53YH e 30F53VYH, (cultivar 1 e 2, respectivamente), não tratadas quimicamente, quando submetidas ao matricondicionamento em diferentes tempos usando-se quatro temperaturas, bem como, na sequência avaliar a qualidade fisiológica destas em função da préhidratação controlada.

\section{Material e Métodos}

O trabalho foi conduzido no Laboratório de Análises de Sementes, da Universidade Tecnológica Federal do Paraná, Câmpus Dois Vizinhos (UTFPR, CâmpusDV).

Foram utilizadas sementes básicas sem tratamento químico de dois lotes de milho híbrido de diferentes vigores, cultivar 1 (30F53YH) e cultivar 2 (30F53VYH). As sementes estavam armazenadas em sacos de papel do tipo $\mathrm{Kraft}^{\circ}$ de dimensão $28 \times 18(\mathrm{~cm})$ em ambiente com temperatura e umidade controlada $\left(10{ }^{\circ} \mathrm{C}\right.$ e $20 \%$ respectivamente). Para caracterização dos lotes, avaliou-se o peso de mil sementes (PMS) de Acordo com as Regras para Análise de Sementes - RAS (BRASIL, 2009). A quantificação do teor de água inicial foi realizada em estufa 105 ${ }^{\circ} \mathrm{C}$, durante 24 horas, com duas repetições de $5 \mathrm{~g}$ por lote.

$\begin{array}{ccc}\text { O experimento foi dividido em duas } \\ \text { partes, } & \text { ambos } & \text { mediante }\end{array}$
matricondicionamento em quatro temperaturas $\left(10,15,2025^{\circ} \mathrm{C}\right)$. A primeira parte do estudo consistiu em determinar o ganho de água de dois lotes de milho descritos acima, para isso utilizouse oito repetições de 200 sementes, acondicionadas em caixas gerbox, contendo vermiculita embebida em água destilada, com $75 \%$ da sua capacidade de retenção. As sementes foram acondicionadas sobre folha de papel Germitest recortada e afixada sobre tela de inox, no interior da caixa, que após tampada, foi lacrada com fita crepe, e levadas para câmaras refrigeradas tipo B.O.D com oito repetições de cada tratamento em uma câmara ajustada na temperatura do respectivo tratamento. As sementes foram pesadas em balança de precisão antes do início da embebição e novamente em intervalos de tempo de $2 ; 4 ; 6 ; 10 ; 14 ; 18$ e 24 
horas (h), acompanhando-se o ganho de massa. Os valores obtidos, em gramas (g), foram transformados para percentagem de água de acordo com a expressão:

$$
\mathrm{U}_{\mathrm{f}}=100-\left[\mathrm{P}_{\mathrm{i}}\left(100-\mathrm{U}_{\mathrm{i}}\right) / \mathrm{P}_{\mathrm{f}}\right]
$$

Em que: $U_{f}$ : umidade final (\%);

$P_{i}$ : peso inicial $(g)$;

$\mathrm{U}_{\mathrm{i}}$ : umidade inicial (\%);

$P_{f}$ : peso final $(g)$.

A segunda parte do experimento, foi realizado após a aplicação do matricondicionamento, sendo estes que formaram o fator temperatura de embebição, em arranjo bifatorial $2 \times 5$ (cultivar $\times$ temperatura de embebição), o delineamento usado foi inteiramente casualizado com quatro repetições de 100 sementes.

Além dos lotes de milho híbrido cultivares (30F53YH e 30F53VYH) testados em ambientes com temperaturas de embebição distintas (10; 15 ; 20 e $25{ }^{\circ} \mathrm{C}$ ) de matricondicionamento, também foram submetidas aos testes de desempenho sementes de ambos lotes sem préhidratação (tratamento controle).

$O$ teste de germinação, utilizado para realizar a primeira contagem de germinação (PCG $\%)$, contagem final de germinação (CFG \%) e classificação do vigor de plântulas (VP \%) foram realizados de acordo com as RAS (BRASIL, 2009). Procedimento análogo foi utilizado para avaliação do comprimento da parte aérea (CPA $\mathrm{cm})$, comprimento de raiz $(C R \mathrm{~cm})$ e massa de matéria seca de plântulas (MS g), (NAKAGAWA, 1999).

Para $O$ índice de velocidade de emergência (IVE), velocidade de emergência (VE dias), o coeficiente de emergência (Cem) e a emergência acumulada (EAcum), conduzido em canteiros com solo, seguiu-se o procedimento proposto por Nakagawa (1999).

Os testes de frio, teste de germinação no frio (TGF) e plantas normais ao teste de frio (PNF) foram conduzidos de acordo com a metodologia descrita por Barros et al., (1999) e pelas RAS (BRASIL, 2009).

A condutividade elétrica massal (CE $\mu \mathrm{S} . \mathrm{cm}^{-1} \cdot \mathrm{g}^{-1}$ ) foi calculada utilizando o método da condutividade em massa (HAESBAERT et al., 2017).

Para as análises dos dados utilizou-se o programa $R$ versão 3.4.1 (R Core Team, 2016), bem como, o teste de Shapiro-Wilk ao nível de $5 \%$ de probabilidade de erro, para verificar o pressuposto de normalidade dos resíduos. Apenas as variáveis da segunda parte, CGF e CR não foram atendidas e, portanto, transformadas segundo Box e Cox (1964), por $\lambda=3$ e $\lambda=2$, respectivamente.

$\mathrm{Na}$ primeira parte do experimento os dados foram submetidos a análise de variância (ANAVA) e seguido de análise de regressão aplicando o modelo que se apresentou significativo.

$\mathrm{Na}$ segunda parte, após as transformações os dados foram submetidos a ANAVA e quando significativos, seguiu-se os testes de comparação de múltiplas médias de Tukey $(\alpha=0,05)$.

\section{Resultados e Discussão}

O cultivar 1 (30F53YH) apresentou massa mais elevada quando comparado com o cultivar 2 $30 F 53 \mathrm{VYH}$ (PMS $=327,5$ e $285,2 \mathrm{~g}$, respectivamente), o teor inicial de água foi de 10,68 e 10,62\% para os cultivares 1 e 2, respectivamente. Ambos cultivares apresentaram sementes uniformes com coeficiente de variação (CV) inferior à $4 \%$ (BRASIL, 2009) (Lote $1 \mathrm{CV}=$ $1,11 \%$ e Lote $2 \mathrm{CV}=0,62 \%$ ).

Verificando a primeira parte do estudo, os valores percentuais de umidade de sementes submetidos ao matricondionamento obtiveram efeito significativo quando comparados os tempos dentro de cada lote de forma isolada (Figura 1). 
Figura 1. Ganho de água do cultivar 30F53YH lote 1 (A) e 30F53VYH lote 2 (B) de sementes de milho híbrido expressos em percentagem de umidade (\%) em função do matricondicionamento em períodos de 2, 4, 6, $10,14,18$ e 24 horas e nas temperaturas de $10,15,20$ e $25^{\circ} \mathrm{C}$.
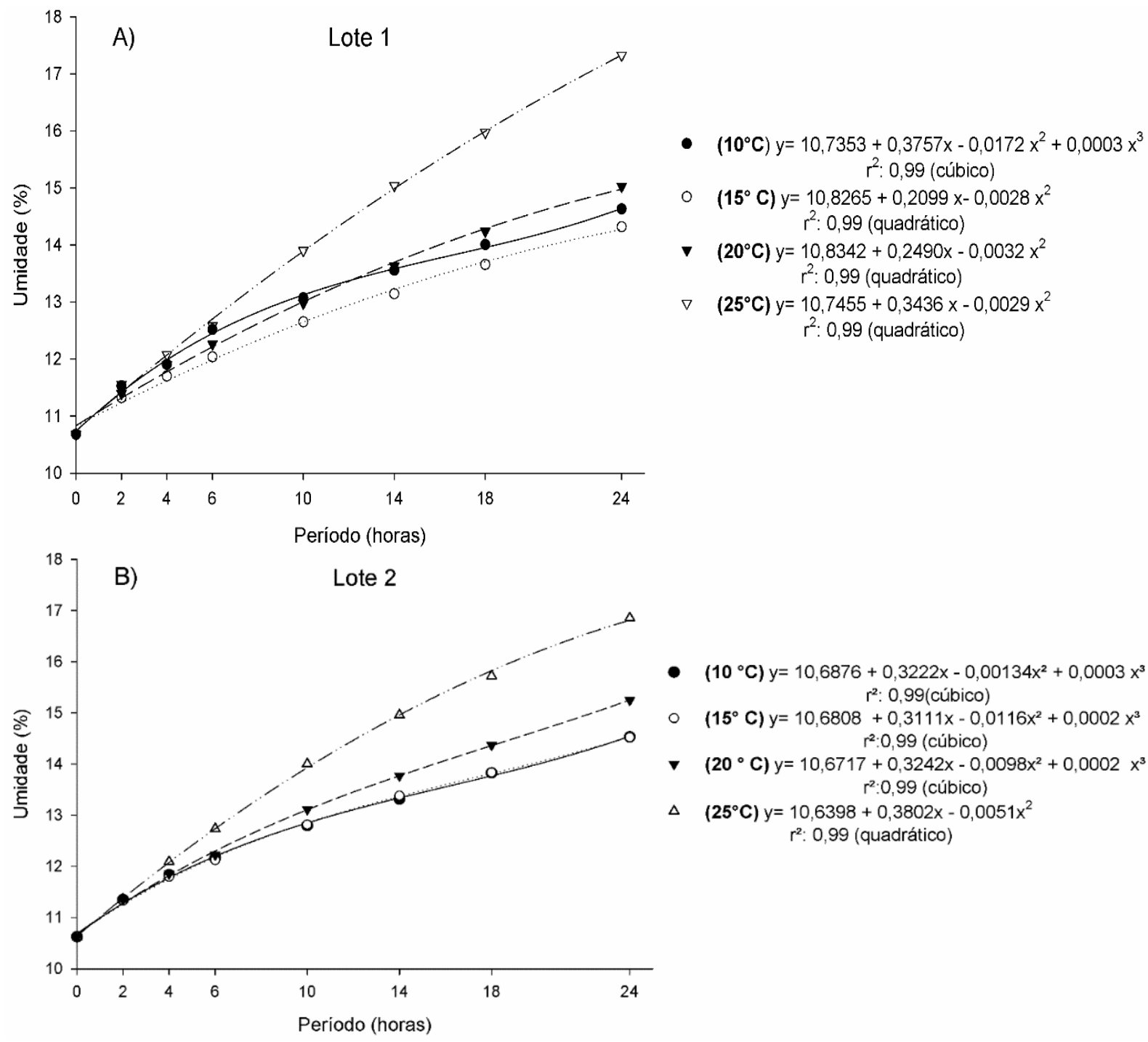

- $\left(10^{\circ} \mathrm{C}\right) \mathrm{y}=10,6876+0,3222 x-0,00134 x^{2}+0,0003 x^{3}$ $r^{2}: 0,99$ (cúbico)

- $\left(15^{\circ}\right.$ C) $y=10,6808+0,3111 x-0,0116 x^{2}+0,0002 x^{3}$ r2:0,99 (cúbico)

V $\left(20^{\circ} \mathrm{C}\right) \mathrm{y}=10,6717+0,3242 \mathrm{x}-0,0098 \mathrm{x}^{2}+0,0002 \mathrm{x}^{3}$ $r^{2}: 0,99$ (cúbico)

$\Delta \quad\left(\mathbf{2 5}^{\circ} \mathrm{C}\right) \mathrm{y}=10,6398+0,3802 \mathrm{x}-0,0051 \mathrm{x}^{2}$ $r^{2}: 0,99$ (quadrático)

Observando-se a primeira fase de germinação com o matricondionamento de sementes de milho em $10{ }^{\circ} \mathrm{C}$, este obteve efeito significativo representado pelo modelo cúbico, e 15,20 e $25^{\circ} \mathrm{C}$ com modelo quadrático para o cultivar 1 com pontos de máxima eficiência técnica (MET) previstos para 38, 39 e 59 horas, respectivamente. A cultivar 2 quando observado as temperaturas de $10,15,20^{\circ} \mathrm{C}$ apresentaramse significativas para o modelo cúbico e na temperatura de $25{ }^{\circ} \mathrm{C}$ o quadrático, com MET previsto para 37 horas.

Assim, as análises de regressão das curvas de cada lote por temperatura, expressam a relação entre o teor de água (\%) dentro dos tempos de embebição. Os dados obtidos neste ensaio sugerem 0 modelo trifásico padrão propostos por Bewley e Black (1978) e Taiz et al. (2017) para evolução do processo de absorção de água pelas sementes. Neste trabalho, caracterizando pelo menos o início da Fase I da germinação de sementes de milho, ao final das 24 horas de avaliação, ambas sementes dos cultivares 30F53YH e 30F53VYH apresentaram maior porcentagem de umidade quando matricondicionadas à temperatura de $25^{\circ} \mathrm{C}$. A maior porcentagem de umidade, em altas temperaturas, pode ser explicada pelo acelerado metabolismo e pela maior permeabilidade do tecido de sementes em temperatura elevada (ULLMANN et al., 2015), além do fato de que altas temperaturas proporcionam saturação de água ao redor das sementes. 
A embebição é a primeira das três etapas que podem culminar na germinação. Nesta fase, o processo geralmente é rápido e ocorre em sementes vivas ou mortas. No entanto, após esta fase, sementes vivas iniciam a Fase II, também conhecida como reativação metabólica (GUIMARÃES et al., 2013; KHATAMI et al., 2015).

No segundo estudo, o cultivar 1 (30F53YH), na PCG (\%) resultados superiores foram obtidos quando as sementes foram submetidas à temperatura de $25^{\circ} \mathrm{C}$ durante $\mathrm{O}$ matricondicionamento, diferindo estatisticamente apenas do controle. Resultado que se manteve na CFG (\%) apenas nas temperaturas de 15 e $20{ }^{\circ} \mathrm{C}$ que mantiveram as maiores médias, não diferindo apenas de sementes matricondicionadas a $10{ }^{\circ} \mathrm{C}$. O cultivar 2 (30F53VYH) apresentou superioridade com as temperaturas de $10,15,20^{\circ} \mathrm{C}$ e Controle $(33,28$, 25 e 30\% respectivamente). A temperatura de 25 ${ }^{\circ} \mathrm{C}$ não favoreceu a PCG, pois a absorção de água em temperatura mais elevada pode ocasionar danos à membrana celular em função da rápida embebição (ZUCARELI et al., 2011). Entretanto, na CFG não foi observada diferença estatística significativa no cultivar 2 dentro das temperaturas testadas, entretanto, foi superior quando comparado com a cultivar 1 em todas temperaturas dentro desta variável (Tabela 1).

Tabela 1. Primeira contagem de germinação (PCG, \%), contagem final de germinação (CFG, \%), plantas normais $(\mathrm{PN}, \%)$, plantas de alto vigor ( $\mathrm{AV}, \%)$, comprimento de raízes de plantas $(\mathrm{CR}, \mathrm{cm})$, massa da matéria seca de plantas (MS, $\mathrm{mg}$ ), de dois lotes de sementes submetidos ao matricondicionamento durante 24 horas e temperaturas de $10,15,20$ e $25^{\circ} \mathrm{C}$, e, tratamento controle. Dados expressos em porcentagem e centímetros

\begin{tabular}{|c|c|c|c|c|c|}
\hline \multicolumn{6}{|c|}{ Primeira Contagem de Germinação - PCG (\%) } \\
\hline & $10^{\circ} \mathrm{C}$ & $15^{\circ} \mathrm{C}$ & $20^{\circ} \mathrm{C}$ & $25^{\circ} \mathrm{C}$ & Controle \\
\hline Cultivar 1 & $24,5 \mathrm{bAB} *$ & $28,0 \mathrm{aAB}$ & $25,0 \mathrm{aAB}$ & 30,5 aA & $19,25 \mathrm{bB}$ \\
\hline Cultivar 2 & 33,75 aA & 28,5 aA & 25,75 aA & $15,5 \mathrm{bB}$ & 30,75 aA \\
\hline CV (\%) & \multicolumn{5}{|c|}{18,3} \\
\hline \multicolumn{6}{|c|}{ Contagem Final de Germinação - CFG (\%) } \\
\hline Cultivar 1 & $53,5 \mathrm{bAB} *$ & $61,25 \mathrm{bA}$ & $63,0 \mathrm{bA}$ & $41,3 \mathrm{bB}$ & $46,75 \mathrm{bB}$ \\
\hline Cultivar 2 & 99,0 aA & 96,0 aA & 99,5 aA & $98,5 \mathrm{aA}$ & $98,0 \mathrm{aA}$ \\
\hline CV (\%) & \multicolumn{5}{|c|}{9,8} \\
\hline \multicolumn{6}{|c|}{ Plantas Normais-PN (\%) } \\
\hline Cultivar 1 & 48,3 bAB* & $56,0 \mathrm{bA}$ & $54,3 \mathrm{bA}$ & $34,8 \mathrm{bB}$ & $40,3 \mathrm{bB}$ \\
\hline Cultivar 2 & $89,3 \mathrm{aBC}$ & $85,5 \mathrm{aC}$ & $96,00 \mathrm{aA}$ & $93,5 \mathrm{aAB}$ & $90,3 \mathrm{aABC}$ \\
\hline $\mathrm{CV}(\%)$ & & & 11,2 & & \\
\hline \multicolumn{6}{|c|}{ Plantas de Alto Vigor - AV (\%) } \\
\hline Cultivar 1 & $15,5 \mathrm{bA}^{*}$ & $21,0 \mathrm{aA}$ & $13,5 \mathrm{bA}$ & $6,8 \mathrm{bA}$ & $16,5 \mathrm{bA}$ \\
\hline Cultivar 2 & $48,5 \mathrm{aC}$ & $23,8 \mathrm{aD}$ & $66,3 \mathrm{aAB}$ & $72,5 \mathrm{aA}$ & $54,5 \mathrm{aBC}$ \\
\hline $\mathrm{CV}(\%)$ & & & & & \\
\hline \multicolumn{6}{|c|}{$\frac{19,2}{\text { Comprimento de Raízes - CR (cm) }}$} \\
\hline Cultivar 1 & $19,8 \mathrm{aA}^{*}$ & 17,4 bBC & $20,2 \mathrm{aA}$ & $18,6 \mathrm{aAB}$ & $15,9 \mathrm{bC}$ \\
\hline Cultivar 2 & $21,0 \mathrm{aA}$ & 19,3 aA & $21,0 \mathrm{aA}$ & $19,9 \mathrm{aA}$ & $20,9 \mathrm{aA}$ \\
\hline CV (\%) & \multicolumn{5}{|c|}{9,7} \\
\hline \multicolumn{6}{|c|}{ Massa da matéria Seca de plantas-MS (mg) } \\
\hline Cultivar 1 & $67,8 \mathrm{bA}^{*}$ & $63,7 \mathrm{bA}$ & $69,7 \mathrm{aA}$ & $71,8 \mathrm{bA}$ & $64,0 \mathrm{bA}$ \\
\hline Cultivar 2 & $84,6 \mathrm{aAB}$ & 82,9 aABC & $71,9 \mathrm{aC}$ & 87,9 aA & 73,6 aBC \\
\hline$* * \mathrm{CV}(\%)$ & \multicolumn{5}{|c|}{7,9} \\
\hline
\end{tabular}


Algumas técnicas de tratamentos prégerminativos podem propiciar melhor desempenho de sementes (BATISTA et al., 2016), tal fato foi observado pelos resultados obtidos no presente trabalho. O matricondicionamento é estratégia de pré-semeadura cujo objetivo é iniciar a atividade metabólica durante o processo de germinação, proporcionando melhor desenvolvimento das plântulas (KHAN et al., 2016). Este método, associado à temperatura de imersão ideal pode proporcionar melhores resultados nos testes de germinação. Binotti et al. (2014), trabalhando com sementes de Brachiaria sp., imersas em solução por período prolongado verificaram que estas têm sua PCG afetada, fato não observado durante a CFG, quando as médias se equipararam estatisticamente. Este fato foi observado também no presente trabalho com o cultivar 2, que apresentou maior CFG em todas as temperaturas testadas, com números superiores comparados ao cultivar 1 devido a qualidade superior das sementes.

A avaliação do vigor de plântulas normais (AV) foi baseada em baixo e alto vigor, mas apenas AV foi demonstrada (Tabela 1). Sementes da cultivar 1 apresentaram valores maiores para $\mathrm{PN}$, quando condicionadas a 10,15 e $20^{\circ} \mathrm{C}$. Isso deve-se ao matricondicionamento em temperaturas ideais que permitiram a embebição dos tecidos sem comprometer sua integridade celular (KHAN et al., 2016). Menores valores de PN podem ser devidos à deterioração e envelhecimento fisiológico das sementes (MATTHEWS; KHAJEH-HOSSEINI, 2007; KHAJEHHOSSEINI et al., 2009).

As sementes provenientes do cultivar 2 apresentaram-se estatisticamente superiores para variável PN quando submetidas ao matricondicionamento em temperaturas de 20 ${ }^{\circ} \mathrm{C}$. Porém, este diferiu apenas das sementes submetidas às temperaturas de 10 e $15{ }^{\circ} \mathrm{C}$. Sementes do cultivar 2 apresentaram valores elevados para PN em qualquer condição de matricondicionamento, quando comparado ao cultivar 1 , atingindo valores superiores a $85 \%$, enquanto o cultivar 1 não apresentou valores maiores que $56 \%$. Tal comportamento também foi apresentado na produção de plântulas de AV (alto vigor), sendo o cultivar 2 superior ao 1.

Ainda na variável $\mathrm{AV}$, a temperatura que proporcionou os melhores resultados foram de
20 e $25{ }^{\circ} \mathrm{C}$, porém, o uso de matricondicionamento não apresentou diferença significativa para esta variável quando observado o cultivar 2. Segundo Marcos Filho (2015), sementes mais vigorosas tendem a apresentar melhor desempenho nos testes fisiológicos. Ambos cultivares apresentaram desenvolvimento uniforme das raízes de plântulas de milho, entretanto, observando o cultivar 1 , foram superiores em temperaturas de 10, $2025^{\circ} \mathrm{C}$ (Tabela 1), sendo estatisticamente iguais.

As plântulas provenientes do cultivar 2 mostraram comprimento de raízes estatisticamente semelhantes quando submetidas à qualquer condição de matricondicionamento e controle. Além disso, os valores foram superiores quando comparados ao cultivar 1.

Para a MS, o cultivar 2 apresentou resultados superiores em quase todas as temperaturas de matricondicionamento, cuja exceção foi na temperatura de $20^{\circ} \mathrm{C}$ (Tabela 1). A MS demonstra que a técnica de matricondicionamento otimiza os processos enzimáticos, proporcionando assim o melhor desenvolvimento (KHAN et al., 2016) e isto ficou evidenciado em algumas das temperaturas testadas.

As sementes do cultivar 1 , além das menores médias de $\mathrm{MS}$, não diferiram estatisticamente entre as temperaturas e controle. Esse comportamento padrão mostra estar diretamente relacionado com o vigor, pois resultou na menor produção de biomassa de plântula, que por sua vez é essencial para o arranque inicial da cultura a campo e estabelecimento.

O cultivar 2, apresentou maior média quando matricondicionadas à $25{ }^{\circ} \mathrm{C}$, mas sem diferir de 10 e $15^{\circ} \mathrm{C}$. A exposição das sementes em ambiente hidratado, indiferente da temperatura, podem ter propiciado ativação das rotas metabólicas e desencadeado o processo germinativo antes do tratamento controle, acarretando no maior desenvolvimento da plântula.

A utilização de técnicas como o matricondicionamento auxilia no acúmulo da massa fresca quando comparada aos tratamentos sem pré-hidratação (KHAN et al., 2016), pois a recuperação das mitocôndrias, com a mais 
adequada forma de hidratação, pode resultar na realização mais precoce do ciclo de Krebs, e consequentemente, na disponibilização mais rápida de maior quantidade de energia capaz de estimular o crescimento e o desenvolvimento das plântulas (GUIMARÃES et al., 2013). Tal fato pode ter ocorrido com o cultivar 2, que apresentou maiores médias em AV e MS.

As variáveis CPA, IVE e TGF apresentaram-se significativas, mas sem interação para os dois fatores testados (cultivar e temperatura). Em CPA, o cultivar 2 obteve maior média com diferença estatística em relação ao cultivar 1. Observando as temperaturas, as sementes matricondicionadas à $25{ }^{\circ} \mathrm{C}$ apresentaram maior média, apesar de diferir apenas do tratamento controle (Tabela 2).

Tabela 2. Comprimento da parte aérea (CPA, cm), teste de germinação no frio (TGF, \%) e índice de velocidade de emergência (IVE) de dois lotes de sementes submetidos ao matricondicionamento durante 24 horas e temperaturas de $10,15,20$ e $25^{\circ} \mathrm{C}$ e, tratamento controle.

\begin{tabular}{lccc}
\hline Lotes & CPA & TGF & IVE \\
\hline Cultivar 1 & $12,2 \mathrm{~b}^{*}$ & $53,5 \mathrm{~b}$ & $10,3 \mathrm{~b}$ \\
Cultivar 2 & $15,1 \mathrm{a}$ & $91,4 \mathrm{a}$ & $15,2 \mathrm{a}$ \\
\hline Temp. ${ }^{\circ} \mathrm{C}$ & & & \\
\hline $10^{\circ} \mathrm{C}$ & $14,0 \mathrm{ab}$ & $72,5 \mathrm{a}$ & $12,4 \mathrm{bc}$ \\
$15^{\circ} \mathrm{C}$ & $13,9 \mathrm{ab}$ & $76,8 \mathrm{a}$ & $13,2 \mathrm{ab}$ \\
$20^{\circ} \mathrm{C}$ & $13,2 \mathrm{ab}$ & $79,3 \mathrm{a}$ & $13,3 \mathrm{a}$ \\
$25^{\circ} \mathrm{C}$ & $14,6 \mathrm{a}$ & $73,5 \mathrm{a}$ & $13,2 \mathrm{ab}$ \\
Controle & $12,7 \mathrm{~b}$ & $51,5 \mathrm{~b}$ & $11,7 \mathrm{c}$ \\
\hline${ }^{* *} \mathrm{CV}(\%)$ & 7,7 & 3,1 & 4,8 \\
\hline
\end{tabular}

*Médias seguidas por letras diferentes, minúsculas na coluna e maiúsculas na linha, diferem pelo teste de Tukey $(p \leq 0,05) .{ }^{* *} \mathrm{CV}$ : coeficiente de variação.

O matricondicionamento propiciou maiores resultados que o tratamento controle para a germinação no teste de frio (TGF) (Tabela 2). Possivelmente, ocorreram algumas alterações químicas e ativação de enzimas pelas diferentes técnicas de matricondicionamento (KHAN et al., 2016), o que explica a elevada germinação quando comparado ao tratamento controle.

Para IVE mais uma vez os resultados foram superiores para o cultivar 2 , o que corrobora por tal cultivar ser mais vigoroso. 0 matricondicionamento nas diferentes temperaturas demonstrou resultados superiores quando aplicados $15,20,25^{\circ} \mathrm{C}$, seguidos de $10{ }^{\circ} \mathrm{C}$ que não diferiram de 15 e $25{ }^{\circ} \mathrm{C}$ e seguido do tratamento controle que foi inferior, mas sem diferir de $10^{\circ} \mathrm{C}$ (Tabela 2).

Temperaturas próximas das ideais para manter as atividades metabólicas permitem emergência mais rápida, afetando as reações bioquímicas que determinam $o$ processo germinativo. Sementes expostas a temperaturas elevadas durante a germinação apresentam melhor índice de velocidade de emergência
(SBRUSSI; ZUCARELI, 2015). Todavia, neste trabalho observou-se que não apenas a temperatura mais alta proporcionou bons resultados, mas também as temperaturas médias para as sementes de milho.

No teste de frio, o número de PNF foi superior no cultivar 2 na maior parte das temperaturas testadas, ao lado de $20^{\circ} \mathrm{C}$, quando foi observada semelhança estatística entre os cultivares (Tabela 3). As sementes do cultivar 1, quando submetidas ao matricondicionamento $20^{\circ} \mathrm{C}$, mostraram resultados superiores as demais temperaturas e controle. Mas quando observado o cultivar 2, os valores superiores foram 10 e $25^{\circ} \mathrm{C}$.

Para os resultados da VE são preferíveis os valores menores, pois indicam menor número de dias necessários para a germinação. No cultivar 1 a temperatura de $25^{\circ} \mathrm{C}$ demonstrou o melhor resultado (Tabela 3) diferindo apenas de $20^{\circ} \mathrm{C}$. No cultivar 2 pode-se observar que o matricondicionamento a temperatura de $20^{\circ} \mathrm{C}$ apresentou bons resultados, mesmo não diferindo das demais, apenas do controle. 
Para Cem, valores superiores são desejáveis. Sementes do cultivar 1 submetidas a temperatura de $25^{\circ} \mathrm{C}$ apresentaram resultados superiores, diferindo apenas de $20^{\circ} \mathrm{C}$ ). $\mathrm{O}$ cultivar 2 a melhor temperatura foi $20^{\circ} \mathrm{C}$, diferindo apenas do controle. Houve diferença estatística entre os cultivares apenas quando as sementes foram submetidas a matricondicionamento em temperatura de $20^{\circ} \mathrm{C}$ onde 0 cultivar 2 apresentou destaque, e sem matricondicionamento onde foram obtidos melhores resultados no cultivar 1 (Tabela 3 ).

Testes de vigor sensíveis a ponto de detectar alterações metabólicas em sua fase inicial são restritos, porém o teste de CE $\left(\mu \mathrm{S} . \mathrm{cm}^{-}\right.$ $\left.{ }^{1} \cdot \mathrm{g}^{-1}\right)$ que está relacionado a integridade da membrana celular, avalia indiretamente o grau de estruturação (COSTA et al., 2008).

Os resultados para CE obtidos com sementes do cultivar 1 submetidas a $25^{\circ} \mathrm{C}$ e cultivar 2 abaixo de $20^{\circ} \mathrm{C}$ foram superiores e diferiram apenas do controle. Houve diferença estatística entre cultivares quando a temperatura utilizada foi de $20^{\circ} \mathrm{C}$, onde o cultivar 2 se destaca, mas no tratamento controle os melhores resultados vieram das sementes do cultivar 1 , em outras temperaturas os resultados foram estatisticamente semelhantes (Tabela 3).

Tabela 3. Plantas normais ao teste de frio (PNF, \%) Velocidade de emergência (VE, \%), coeficiente de emergência e condutividade elétrica $\left(C E,\left(\mu . \mathrm{cm}^{-1} . \mathrm{g}\right)\right.$ de dois lotes de sementes de milho híbrido submetidos a 24 horas de matricondicionamento em temperaturas de $10,15,20$ e $25^{\circ} \mathrm{C}$ e tratamento controle.

\begin{tabular}{|c|c|c|c|c|c|}
\hline \multicolumn{6}{|c|}{ Plantas Normais ao teste de Frio - PNF (\%) } \\
\hline & $10^{\circ} \mathrm{C}$ & $15^{\circ} \mathrm{C}$ & $20^{\circ} \mathrm{C}$ & $25^{\circ} \mathrm{C}$ & Controle \\
\hline Cultivar 1 & $26,5 \mathrm{bB} *$ & $29,3 \mathrm{bB}$ & $48,5 \mathrm{aA}$ & $31,9 \mathrm{bB}$ & $21,7 \mathrm{bB}$ \\
\hline Cultivar 2 & $80,2 \mathrm{aAB}$ & $68,9 \mathrm{aB}$ & $70,6 \mathrm{aB}$ & 81,9 aA & $51,2 \mathrm{aB}$ \\
\hline CV (\%) & \multicolumn{5}{|c|}{5,1} \\
\hline \multicolumn{6}{|c|}{ Velocidade de Emergência - VE } \\
\hline Cultivar 1 & $7,3 \mathrm{aAB}^{*}$ & $7,5 \mathrm{aAB}$ & 7,7 aA & $6,9 \mathrm{aB}$ & $7,1 \mathrm{bAB}$ \\
\hline Cultivar 2 & $7,5 \mathrm{aAB}$ & $7,3 \mathrm{aAB}$ & $7,00 \mathrm{bB}$ & $7,19 \mathrm{aAB}$ & $7,8 \mathrm{aA}$ \\
\hline CV (\%) & & & 4,9 & & \\
\hline \multicolumn{6}{|c|}{ Coeficiente de emergência - Cem } \\
\hline Cultivar 1 & $13,8 a \mathrm{AB}^{*}$ & $13,3 \mathrm{aAB}$ & $12,9 \mathrm{bB}$ & 14,5 aA & $14,2 \mathrm{aAB}$ \\
\hline Cultivar 2 & $13,3 \mathrm{aAB}$ & $13,7 \mathrm{aAB}$ & 14,4 aA & $14,1 \mathrm{aAB}$ & $12,9 \mathrm{bB}$ \\
\hline CV (\%) & & & 5,1 & & \\
\hline \multicolumn{6}{|c|}{ Condutividade Elétrica - CE $\left(\mu . \mathrm{cm}^{-1} . \mathrm{g}\right)$} \\
\hline Cultivar 1 & $0,3 a A^{*}$ & $0,3 \mathrm{aAB}$ & $0,2 \mathrm{aB}$ & $0,2 \mathrm{aB}$ & $0,3 \mathrm{aAB}$ \\
\hline Cultivar 2 & $0,1 \mathrm{bA}$ & $0,1 \mathrm{bA}$ & $0,1 \mathrm{bA}$ & $0,1 \mathrm{bA}$ & $0,2 \mathrm{bA}$ \\
\hline${ }^{* *} \mathrm{CV}(\%)$ & & & 12,39 & & \\
\hline
\end{tabular}

*Médias seguidas por letras diferentes, minúsculas na coluna e maiúsculas na linha, diferem pelo teste de Tukey $(p \leq 0,05) .{ }^{*} C V$ : coeficiente de variação.

Os valores de CE obtidos no cultivar 1 foram superiores ao cultivar 2 em todos os níveis de temperatura (Tabela 3). O processo de embebição pode causar danos às sementes, apresentando resultados de condutividade elétrica de modo que quanto maior o dano, maior a quantidade de solutos lixiviados (FRANCO ROSA et al., 2000). Para os autores, um processo de reidratação mais lento favorece os sistemas de membranas recuperem a sua estrutura lamelar, de maneira que, quando imersas em água, permitissem menor lixiviação de solutos. 
Isto significa que maiores leituras de condutividade apresentam sistemas de membranas mais danificadas, o que pode ter ocorrido com o cultivar 1, ou talvez o consumo de reserva própria para se manter viável.

Sementes vigorosas tendem a demonstrar melhor desenvolvimento durante os testes fisiológicos (MARCOS FILHO, 2015), o que foi observado no presente trabalho de maneira geral é que, as análises das variáveis como CFG, PN, AV, MS, CPA, TGF, IVE e PNF foram superiores quando analisadas o cultivar 2, considerando tais característica como de melhor vigor. Entretanto,

o uso de matricondicionamento favoreceu ambos cultivares; aliás, o cultivar 1 que de maneira geral é inferior, obteve aumento significativo em qualidade fisiológica com aplicação de pré-embebição controlada como exemplo resultados de, PCG, CFG, PN, CPA, TGF, CE.

A técnica de condicionamento de matrizes é responsável por otimizar processos enzimáticos proporcionando melhor desenvolvimento (KHAN et al., 2016) e isso permaneceu eminente em algumas das temperaturas testadas.

O teste de vigor é capaz de detectar pequenas alterações metabólicas na fase inicial da germinação; porém, a CE está relacionada à integridade da membrana (COSTA et al., 2008). A técnica pode causar danos estruturais e pode ser detectado pelo teste CE e quanto maior o dano, maiores valores são obtidos em função dos solutos lixiviados para solução (ULLMANN et al., 2015).

Figura 2. Emergência acumulada de dois cultivares de sementes de milho, cultivar 1 (A), cultivar 2 (B) submetidas ao matricondicionamento por 24 horas em temperaturas de $10{ }^{\circ} \mathrm{C}, 15{ }^{\circ} \mathrm{C}, 20{ }^{\circ} \mathrm{C}$ e $25{ }^{\circ} \mathrm{C}$ e tratamento controle.
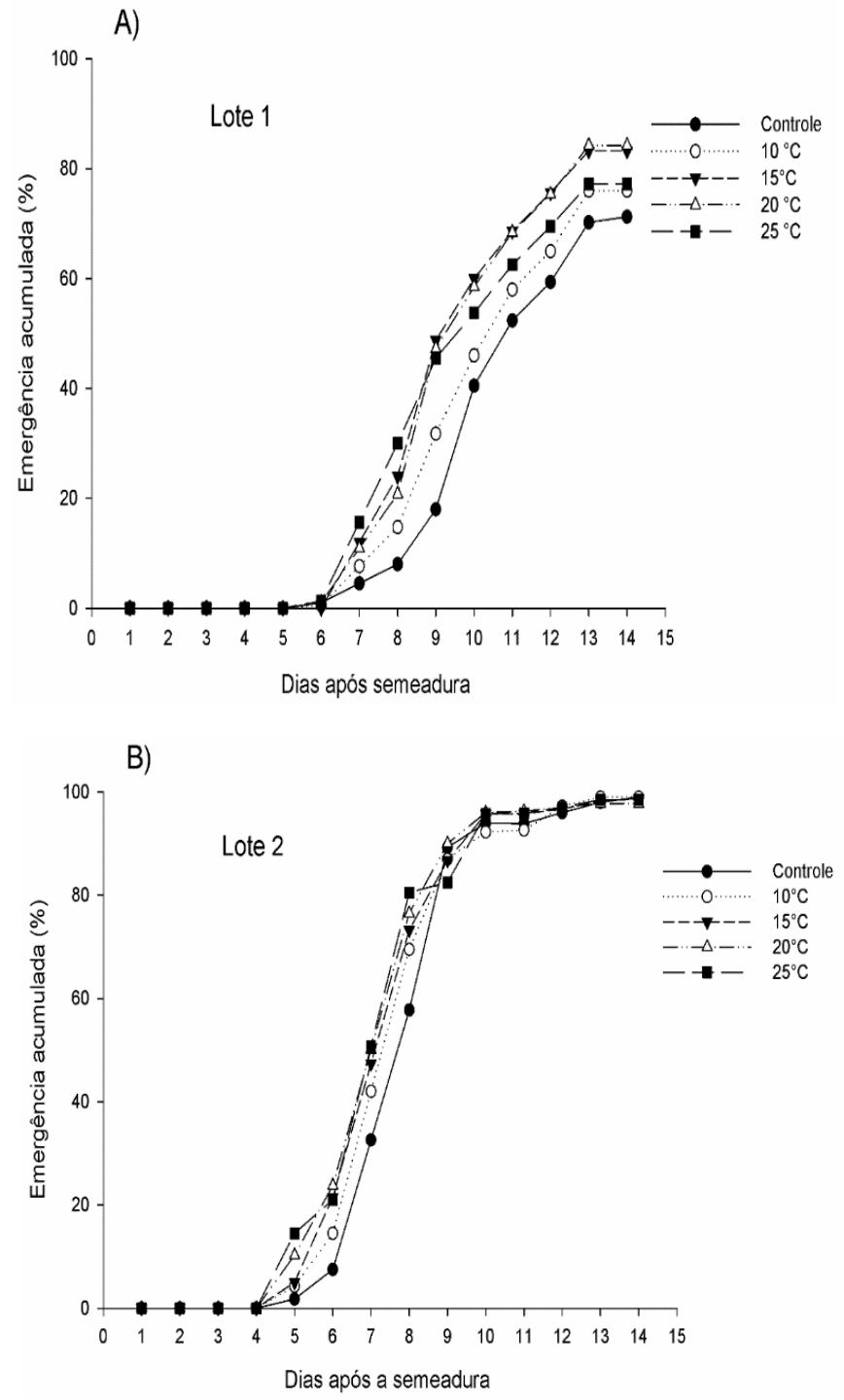
A emergência acumulada (EA), o cultivar 2 apresentou maiores resultados que o cultivar 1 independente da temperatura (Figura 2). A germinação ocorre a partir do $5^{\circ}$ dia e estabilizou-se no $10^{\circ}$ dia após a semeadura, o que já seria esperado quando verificado os resultados de testes de qualidades fisiológica feitos em laboratório. Quando as sementes de milho do cultivar 2 foram submetidas ao método de matricondicionamento, a EA foi favorecida de acordo com o aumento de temperatura de embebição. 0 mesmo pode ter ocorrido para o cultivar 1 que com o uso da pré-embebição controlada pode ter contribui com a manutenção celular e ocasionado melhor EA.

A menor germinação das sementes é determinada por vários fatores, incluindo a qualidade das sementes e os fatores ambientais com temperaturas próximas ao ideal, assim permite rápida emergência, afeta a reação bioquímica durante a germinação (KHAN et al., 2016), os dados corroboram com está afirmação, pois, a qualidade fisiológica foi superior no cultivar 2; porém, houve aumento de qualidade no cultivar inferior, quando aplicado a técnica de matricondicionamento.

Portanto, quando aplica-se técnicas de pré-embebição, a germinação ocorre, e o aumento de temperatura facilita a permeabilidade da membrana e maior captação de água, que por sua vez, diminuem níveis de oxigênio no espaço intracelular, levando à redução de produção de energia e retardando o processo de germinação (GUIMARÃES et al., 2013); por isso, é importante o controle de entrada de água influenciado pela temperatura.

Os mecanismos moleculares de proteção de membranas podem ser ativados durante tratamentos de pré-embebição, diminuindo a lixiviação do conteúdo celular e garantindo a compartimentalização celular necessária permitindo melhor desempenho durante a germinação (COSTA et al., 2008), pois com o uso da técnica apresentou melhores resultados de germinação.

O que ocorreu para o cultivar 2, apresentar maiores resultados desde a germinação (Tabela 1), bem como, os menores valores encontrados na condutividade elétrica (Tabela 3), indicando que a integridade das células fora mantida, possivelmente pela melhor qualidade inicial do lote deste cultivar.
Sementes inferiores, com menor potencial fisiológico, apresentam ausência de proteção na membrana durante a hidratação inicial da fase I, levando a diminuição de vigor e germinação (COSTA et al., 2008), podendo ser este fato a explicação para as observações dos resultados referentes ao cultivar 1 .

Observando todos os resultados obtidos, pode-se observar bons resultados superiores quando as sementes são submetidas a matricondicionamento ou provenientes de lotes com maior vigor.

\section{Conclusão}

Os resultados permitiram concluir que a qualidade fisiológica das sementes de milho foi afetada pela hidratação controlada e que as maiores temperaturas de embebição proporcionam a expressão de maior vigor nas plântulas de milho. A técnica do matricondicionamento se mostrou eficaz para realizar o condicionamento osmótico de sementes de milho.

\section{Referências}

BARROS, A. S. R.; DIAS, M. C. L. L.; CICERO, S. M; KRZYZANOWSKI. F. C. "Teste de frio". In: KRZYZANOWSKi, F. C.; VIEIRA, R. D.; FRANÇA NETO, J. B. (Ed.). Vigor de sementes: conceitos e testes. Londrina: ABRATES, 1999. p. 5-15.

BATISTA, T. B.; BINOTTI, F. F. D. S.; CARDOSO, E. D.; COSTA, E.; NASCIMENTO, D. M. D. Appropriate hydration period and chemical agent improve priming in brachiaria seeds. Pesquisa Agropecuária Tropical, v. 46, n. 3, p. 350-356, $2016 . \quad$ http://dx.doi.org/10.1590/1983$40632016 v 4638422$.

BEWLEY, J. D.; BLACK, M. Physiology and biochemistry of seed in relation to germination. Berlin: Springer Verlag, 1978. $306 \mathrm{p}$. https://doi.org/10.1007/978-3-642-66668-1

BINOTTI, F. F. S.; SUEDA JÚNIOR, C. I.; CARDOSO, E. D.; HAGA, K. I.; NOGUEIRA, D.C. Tratamentos pré-germinativos em sementes de Brachiaria. Agrária - Revista Brasileira de Ciências Agrárias, v. 9, n. 4, p. 614-618, 2014.

https://doi.org/10.5039/agraria.v9i4a2781.

BOX, G. E. P.; COX, D. R. An analysis of 
transformations. Journal of the royal statistical society, v. 26, n. 2, p. 211-252, 1964.

https://doi.org/10.1111/j.2517-

6161.1964.tb00553.x

BRASIL. Ministério da Agricultura e Reforma Agrária. Secretaria Nacional de Defesa Agropecuária. Regras para análise de sementes. Brasília, DF: SDA, 2009. 398 p.

CARVALHO, N. M.; NAKAgAWA, J. Sementes: ciência, tecnologia e produção. 4. ed. Jaboticabal: FUNEP, 2000. $588 \mathrm{p}$.

COSTA, C. J.; VILLELA, F. A.; BERTONCELLO, M. R.; TILLMANN, M. A. A.; MENEZES, N. L. Préhidratação de sementes de ervilha e sua interferência na avaliação do potencial fisiológico. Revista Brasileira de Sementes, v. 30, n. 1, p. 198-207, 2008.

http://dx.doi.org/10.1590/S0101-

31222008000100025.

DIAS, L. B. X.; QUEIROZ, P. A. M.; FERREIRA, L. B. S.; SANTOS, W. V.; FREITAS, M. A. M.; SILVA, P. P.; NASCIMENTO, W. M.; LEÃO-ARAÚJO, E. F. Teste de condutividade elétrica e embebição de sementes de grão-de-bico. Brazilian Journal of Agricultural Sciences/Revista Brasileira de Ciências Agrárias, v. 14, n. 2, p. 1-8, 2019.

https://doi.org/10.5039/agraria.v14i2a5641

FRANCO ROSA, S. D. V. da; PINHO, R. V.; VIEIRA, M. G. G. C.; VEIGA, R. D. Eficácia do teste de condutividade elétrica para uso em estudos de danos de secagem em sementes de milho. Revista Brasileira de Sementes, v. 22, n. 1, p. 5463, 2000. https://doi.org/10.17801/01013122/rbs.v22n1p54-63

FREITAS, R. A.; NASCIMENTO, W. M. Accelerated aging test on lentil seeds. Revista Brasileira de Sementes, v. 28, n. 3, p. 59-63, 2006.

http://www.scielo.br/pdf/rbs/v28n3/09. https://doi.org/10.1590/S0101-

31222006000300009

GRZYBOWSKI, C. R. S.; VIEIRA, R. D.; PANOBIANCO, M. Testes de estresse na avaliação do vigor de sementes de milho. Revista Ciência Agronômica, v. 46, n. 3 p. 590-596, 2015.

http://www.scielo.br/pdf/rca/v46n3/0045-6888rca-46-03-0590.pdf.
GUIMARÃES, M. A.; TELLO, J. P. J.; DAMASCENO, L. A.; VIANA, C. S.; MONTEIRO, L. R. Préembebição de sementes e seus efeitos no crescimento e desenvolvimento de plântulas de melancia. Revista Ceres, v. 60, n. 3, p. 442-446. $2013 . \quad$ https://doi.org/10.1590/S0034$737 \times 2013000300020$

HAESBAERT, F. M.; LOPES, S. J.; MERTZ, L. M.; LÚCIO, A. D.; HUTH, C. Tamanho de amostra para determinação da condutividade elétrica individual de sementes de girassol. Bragantia, $v$. 76, n. 1, p. 54-61, 2017.

https://doi.org/10.1590/1678-4499.389

KHAJEH-HOSSEINI, M.; LOMHOLT, A.; MATTHEWS, S. "Mean germination time in the laboratory estimates the relative vigour and field performance of commercial seed lots of maize (Zea mays L.)." Seed Science and Technology, v. 37, n. 2, p. 446-456, 2009.

https://doi.org/10.15258/sst.2009.37.2.17

KHAN, A. Z.; IMRAN, M. A.; KHALIL, A.; GUL, H.; AKBAR, H.; WAHAB, S. Impact of Fertilizer Priming on Seed Germination germination behavior and vigor of maize. International Society of Pure and Applied Biology, v. 5, n. 4, p. 183-192, 2016.

http://dx.doi.org/10.19045/bspab.2016.50024

KHAN, A. A.; MIURA, H.; PRUSINKI, J.; ILYAS, S. Matriconditioning of seed to improve emmergence. Minneapolis: Procedings of The Symposium on Stand Establishment of Horticultural Crops, 1990. 26 p.

KHAN, A. A.; JAMES D. M.; GEORGE, S. A.; SATRYAS, I. Matriconditioning of vegetable seeds to improve stand establishment in early field plantings. Journal American Society Horticulture Science, v. 117, n. 1, p. 41-47, 1992.

https://doi.org/10.21273/JASHS.117.1.41

KHATAMI, S. R.; SEDGHI, M.; SHARIFI, R. S. Influence of priming on the physiological traits of corn seed germination under drought stress. Annals of West University of Timişoara, Series of Biology, v. 18, n. 1, p. 1-6, 2015.

https://biologie.uvt.ro/annals/vol_18_1/AWUTSe rBio_June2015_1-6.pdf.

MARCOS FILHO, J. Seed vigor testing: an overview 
of the past, present and future perspective. Scientia Agricola, v. 72, n. 4, p. 363-374, 2015. https://doi.org/10.1590/0103-9016-2015-0007

MARTINS, C. C.; MACHADO, C. G.; CAVASINI, R. Temperatura e substrato para o teste de germinação de sementes de pinhão-manso. Ciência e Agrotecnologia, v. 32, n. 3, p. 863-868, $2008 . \quad$ https://doi.org/10.1590/S141370542008000300024

MATTHEWS, S.; KHAJEH-HOSSEINI, M. Length of the lag period of germination and metabolic repair explain vigour differences in seed lots of maize (Zea mays). Seed Science and Technology, v. 35, n. 1, p. 200-212, 2007.

https://doi.org/10.15258/sst.2007.35.1.18.

NAKAGAWA, J. Testes de vigor baseados no desempenho de plântulas. In: KRZYZANOWSKI, F. C.; VIEIRA, R. D.; FRANÇA NETO, J. B. (Ed.). Vigor de sementes: conceitos e testes. São Paulo: ABRATES, 1999. p. 2-24.

PALLAORO, D. S.; CAMILI, E. C.; GUIMARAES, S.; ALBUQUERQUE, M. C. F. Methods for priming maize seeds. Journal of Seed Science, v. 38, n. 2, p. 148-154, 2016. https://doi.org/10.1590/2317$1545 v 38 n 2161132$

PASSOS, M. A. A.; SILVA, F. J. B. C.; SILVA, E. C. A.; PESSOA, M. M. L.; SANTOS, R. C. Luz, substrato e temperatura na germinação de sementes de cedro-vermelho. Pesquisa Agropecuária Brasileira, v. 43, n. 2, p. 281-284, 2008.

http://dx.doi.org/10.1590/S0100204X2008000200019.

$\mathrm{R}$ Core Team. A language and environment for statistical computing. Vienna: $R$ Foundation for Statistical Computing, 2016. Disponível em: https://www.r-project.org/. Acesso em: 03 ago.
2015.

SBRUSSI, C. A. G.; ZUCARELI, C. Germinação sob altas temperaturas para avaliação do potencial fisiológico de sementes de milho. Ciência Rural, v. 45, n. 10, p. 1736-174, 2015.

http://dx.doi.org/10.1590/01038478 cr20130906.

SCHEEREN, B. R.; PESKE, S. T.; SCHUCH, L. O. B.; BARROS, A. C. A. Qualidade fisiológica e produtividade de sementes de soja. Revista Brasileira de Sementes, v. 32, n. 3, p. 35-41, 2010. https://doi.org/10.1590/S0101-

\section{4}

TAIZ, L., ZEIGER, E., MOLLER, I. M., MURPHY, A. Fisiologia e desenvolvimento vegetal. 6. ed. Porto Alegre: Artmed, 2017. 858 p.

TIAN, Y.; GUAN, B.; ZHOU, D.; YU, J.; LI, G.; LOU, $Y$. Responses of seed germination, seedling growth, and seed yield traits to seed pre treatment in maize (Zea mays L.). The Scientific World Journal, p. 1-8. 2014. http://dx.doi.org/10.1155/2014/834630.

ULLMANN, R.; RESENDE, O.; CHAVES, T. H.; OLIVEIRA, D. E. C.; COSTA, L. M. Qualidade fisiológica das sementes de sorgo sacarino submetidas à secagem em diferentes condições de ar. Revista Brasileira de Engenharia Agrícola e Ambiental, v. 19, n. 1, p. 64-69, 2015.

http://dx.doi.org/10.1590/18071929/agriambi.v19n1p64-69.

ZUCARELI, C.; CAVARIANI, C.; OLIVEIRA, E. A. P.; NAKAGAWA, J. Métodos e temperaturas de hidratação na qualidade fisiológica de sementes de milho. Revista Ciência Agronômica, v. 42, n. 3, p. 684-692, 2011.

http://ccarevista.ufc.br/seer/index.php/ccarevist a/article/view/1420. 